Article

\title{
Interface Interactions in Conjugated Polymer Composite with Metal Oxide Nanoparticles
}

\author{
Tsegaye Belege Atisme, Chin-Yang Yu ${ }^{\mathbb{D}}$, Eric Nestor Tseng $\mathbb{D}^{\mathbb{D}}$, Yi-Che Chen, Pei-Kai Hsu and \\ Shih-Yun Chen *
}

Department of Materials Science and Engineering, National Taiwan University of Science and Technology, 43, Section 4, Keelung Road, Taipei 10607, Taiwan; tsfre99@gmail.com (T.B.A.); cyyu@mail.ntust.edu.tw (C.-Y.Y.); ernestor88@gmail.com (E.N.T.); d10704001@mail.ntust.edu.tw (Y.-C.C.); D10804002@mail.ntust.edu.tw (P.-K.H.)

* Correspondence: SYChen@mail.ntust.edu.tw

Received: 28 September 2019; Accepted: 28 October 2019; Published: 29 October 2019

check for updates

\begin{abstract}
This study presents the preparation, characterization, and properties of a new composite containing cerium oxide nanoparticles and a conjugated polymer. $\mathrm{CeO}_{2}$ nanoparticles prepared using the co-precipitation method were dispersed into the conjugated polymer, prepared using the palladium-catalyzed Suzuki-Miyaura cross-coupling reaction. The interface interactions between the two components and the resultant optoelectronic properties of the composite are demonstrated. According to transmission electron microscopy and X-ray absorption spectroscopy, the dispersion of $\mathrm{CeO}_{2}$ nanoparticles in the polymer matrix strongly depends on the $\mathrm{CeO}_{2}$ nanoparticle concentration and results in different degrees of charge transfer. The photo-induced charge transfer and recombination processes were studied using steady-state optical spectroscopy, which shows a significant fluorescence quenching and red shifting in the composite. The higher photo-activity of the composite as compared to the single components was observed and explained. Unexpected room temperature ferromagnetism was observed in both components and all composites, of which the origin was attributed to the topology and defects.
\end{abstract}

Keywords: dispersion; charge-transfer; fluorescence quenching; red-shifting; defects

\section{Introduction}

Composites containing nanoparticles have received much attention due to their synergistic and hybrid properties derived from their corresponding components. The composite properties depend on the properties of their individual components and also on their morphologies and interfacial characteristics. For instance, Bellucci et al. demonstrated that the optical properties changes were correlated to nanoparticle-driven interface modulations [1]. The potential uses of composites include battery cathodes and solid-state ionics [2,3], supercapacitor and dielectrics [4,5], catalysts [6,7], corrosion protection [8,9], sensors [10], optical and electrical materials [11,12], ultraviolet (UV) blocking [13-15] and many others. In these recent years, composites with the use of organic-inorganic hybrids were found to possess improved and new properties. The main challenge in polymer-inorganic nanoparticles (NP) is cluster or agglomerate formation due mainly to the presence of strong Van Der Waals and electrostatic forces. In general, there are two preparative methods for these composites such as the in situ and the ex situ methods [16]. The in situ method prepares the nanoparticles in the polymer solution or monomer polymerization containing the nanoparticles. This method gives better nanoparticle dispersion but the problem is nanoparticle contamination with solids that should be removed. The ex situ method involves nanoparticle and polymer preparation separately before mixing them in a solvent using mechanical forces. This method is relatively simple and commonly used for large-scale 
production even if the dispersion is less efficient compared to the in situ method and difficult to maintain stable dispersion.

Among the rare earth metal oxides that have been used for composite fabrication, cerium (IV) oxide (ceria, $\mathrm{CeO}_{2}$ ) is one of the most used metal oxides because cerium is the most abundant element among the rare earths and is environmentally friendly [17]. An interesting property of $\mathrm{CeO}_{2}$ is that it can have a stable structure far from the stoichiometric proportions of oxygen. The relaxation or reconstruction of materials with defects at high concentrations is unusual. Both in theory and experimentally, it has been shown that the defects tend to concentrate at the surface, which leads to unique catalytic behavior as well as magnetism. T. Masui et al. worked the relaxatived auto-dispersion (RAD) process to prepare $\mathrm{CeO}_{2}$ nanoparticles dispersed in Nylon 11 film matrix [18]. They prepared $\mathrm{CeO}_{2}$ nanoparticles with an average size of $5 \mathrm{~nm}$. The uniform dispersion of $\mathrm{CeO}_{2}$ nanoparticles in the polymer matrix was observed by the preparation of $\mathrm{CeO}_{2}$ from ultrafine cerium metal particles deposited upon nylon film in vacuum using oxidation in air. W. Wang et al. used a direct facile synthetic strategy which is the in situ type to incorporate $\mathrm{CeO}_{2}$ in regenerated cellulose films [19]. In their work, $\mathrm{CeO}_{2}$ was fairly uniformly dispersed in the polymer matrix with an average size of about $24 \mathrm{~nm}$. When the $\mathrm{CeO}_{2}$ precursor concentration increased, the $\mathrm{CeO}_{2}$ nanoparticle agglomeration in the polymer also became higher. Their work showed that the prepared nanocomposites have moderate thermal stability, a certain degree of hydrophobicity and desired UV-shielding property. However, this method may not be suitable to control the nanoparticle shape and size.

Recently, F. C. da Silva et al. further demonstrated the charge transfer process between $\mathrm{CeO}_{2}$ and 1,4,5,8-naphthalenediimide [20]. In their work, they briefly explained that the photochemical property of the diimide molecules in the nanocomposites can be tuned at different $\mathrm{pH}$ values due to the sorption of molecules on the $\mathrm{CeO}_{2}$ nanoparticle surface. The diimide molecules adsorb onto the surface and are sensitive to the $\mathrm{CeO}_{2}$ surface charge. In more acidic conditions, the fluorescence channel of the naphthalenediimide is triggered, while at basic condition, the creation of charge transfer complexes is induced. Thus, finding a suitable technique for synthesizing a composite in which $\mathrm{CeO}_{2}$ nanoparticles are dispersed homogeneously in a polymer matrix is a fascinating task. Tuning the properties of the composites is necessary to investigate the interactions between polymers and oxides in more detail.

In this paper, a conjugated polymer containing 2,7-linked spirobiflourene and naphthalene bisimide was utilized to form a composite with $\mathrm{CeO}_{2}$ nanoparticles. An ex situ method was used to prepare the polymer composite with $\mathrm{CeO}_{2}$ nanoparticles. The polymer used in this study can accept electron from electrophilic species. On the other hand, $\mathrm{CeO}_{2}$ is a superior catalyst, which easily adjusts the surface condition. Nanoparticle dispersion, interactions between the components, structure and the physical properties of the composites are investigated and compared with that of the individual components.

\section{Experimental Procedure}

\subsection{Synthesis of Polymer}

The conjugated polymer was synthesized by the modification procedures reported previously [21]. All chemicals and reagents were purchased from commercial sources (TCI, Alfa Aesar (Haverhill, MA, United States) or Sigma Aldrich (St. Louis, MO, United States)) and used without further purification unless otherwise noted. The monomers are 2,7-bis(4,4,5,5-tetramethyl-1,3,2-dioxaborolan-2-yl)-9,9'spirobifluorene, 4,7-dibromobenzo[c] thiadiazole and $N, N^{\prime}$-bis(2-ethylhexyl)-2,6-dibromonaphthalene1,4,5,8-tetracarboxylic acid diimide. Other chemicals used for polymer synthesis are aqueous potassium carbonate $\left(\mathrm{K}_{2} \mathrm{CO}_{3}\right)$, tetrakis (triphenylphosphine) palladium and 1,4-dioxane. This alternating copolymer was obtained via Suzuki-Miyaura cross coupling reaction of one equivalent of 4,7-dibromobenzo[c] thiadiazole, two equivalents of 2,7-bis(4,4,5,5-tetramethyl-1,3,2-dioxaborolan2-yl)-9,9'-spirobifluorene and one equivalent of $N, N^{\prime}$-bis(2-ethylhexyl)-2,6-dibromonaphtalene-1,4,5,8tetracarboxylic acid diimide. 2,7-Bis(4,4,5,5-tetramethyl-1,3,2-dioxaborolan-2-yl)-9,9'-spirobifluorene 
(0.114 g, $0.20 \mathrm{mmol}), \quad$ 4,7-dibromobenzo[c] thiadiazole $(0.030 \mathrm{~g}, \quad 0.10 \quad \mathrm{mmol})$, N,N'-bis(2-ethylhexyl)-2,6-dibromonaphtalene-1,4,5,8-tetracarboxylic acid diimide (0.065 g, $0.10 \mathrm{mmol})$ and aqueous $\mathrm{K}_{2} \mathrm{CO}_{3}(2 \mathrm{M}, 4.0 \mathrm{~mL})$ were mixed in 1,4-dioxane $(20 \mathrm{~mL})$. After degassing for $10 \mathrm{~min}$, tetrakis(-triphenylphosphine) palladium $(0.012 \mathrm{~g}, 5 \mathrm{~mol} \%)$ was added under an argon atmosphere. The reaction mixture was heated at $110{ }^{\circ} \mathrm{C}$ for $48 \mathrm{~h}$ before cooling to room temperature. The reaction mixture was extracted with dilute aqueous hydrochloric acid and dichloromethane. The organic layers were combined, washed with brine, dried over anhydrous magnesium sulfate. After filtration, the residue was concentrated and poured into methanol and then the precipitate was collected by filtration. The solid was washed by a Soxhlet extraction with methanol and acetone for $24 \mathrm{~h}$, respectively before being dissolved in hot chloroform. The product was dried under a vacuum to give a yield of $65 \%$. The detailed synthetic process of the polymer is shown in Figure S1. More details of the polymerization reaction condition and the structure characterization of the polymer are reported in reference [21].

\subsection{Synthesis of Ceria Nanoparticles}

Cerium (III) nitrate hexahydrate $\left(\mathrm{Ce}\left(\mathrm{NO}_{3}\right)_{3} \cdot 6 \mathrm{H}_{2} \mathrm{O} ; 434.23 \mathrm{~g} / \mathrm{mol}, 99.5 \%\right.$ purity) was purchased from Alfa Aesar. Ammonium hydroxide $\left(\mathrm{NH}_{4} \mathrm{OH} ; 35.05 \mathrm{~g} / \mathrm{mol}, 20 \%-30 \%\right)$ was purchased from Avantor (Radnor, PA, United States). Ethylene glycol (99.5\% purity) was purchased from Sigma-Aldrich. These chemicals were used without further purification. Distilled water was used in the synthesis process for nanoparticles.

$\mathrm{CeO}_{2}$ nanoparticles were synthesized by the co-precipitation method. The $\mathrm{Ce}\left(\mathrm{NO}_{3}\right)_{3} \cdot 6 \mathrm{H}_{2} \mathrm{O}$ is dissolved in $80 \%$ ethylene glycol (EG)/water mixture by stirring at $600 \mathrm{rpm}$ at room temperature. When all the nitrate precursors are dissolved, ammonium hydroxide $(3 \mathrm{M})$ is added dropwise while the solution is kept in the same condition. After the addition of the precipitating agent (i.e., $\mathrm{NH}_{4} \mathrm{OH}$ ), the mixture was stirred at $30{ }^{\circ} \mathrm{C}$ for $21 \mathrm{~h}$. The precipitate was separated by centrifugation at $6000 \mathrm{rpm}$ for $10 \mathrm{~min}$ and washed three times with ethanol. The separated solid was dried for $24 \mathrm{~h}$ and crushed with mortar and pistil, the $\mathrm{CeO}_{2}$ nanoparticles were obtained as powders.

\subsection{Synthesis of $\mathrm{CeO}_{2} /$ Polymer Nanoparticle (NP) Composites}

Polymer is dissolved in tetrahydrofuran (THF) with a concentration of $0.001 \mathrm{~g} / \mathrm{mL}$ and sonicated for 30 min. Different amounts of $\mathrm{CeO}_{2}$ nanoparticle powders with a 20, 40 and 50 weight percent were added into the polymer solution and the mixture was sonicated for $30 \mathrm{~min}$. The composites were left in the oil bath for $72 \mathrm{~h}$.

\subsection{Characterization}

The NPs were characterized by using the X-ray diffractometer (XRD) with $\mathrm{Cu}$ K $\alpha$ radiation and beamline 01C2 at the National Synchrotron Radiation Research Center (NSRRC), Taiwan. The particle distribution, morphology, and crystal structure are studied by a transmission electron microscope operated at $200 \mathrm{keV}$ (Philips Technai G2 FEI-TEM) (Thermo Fisher Scientific Co. Amsterdam, Netherlands). The ultraviolet-visible (UV-Vis) spectra were recorded using a Jasco V-670 spectrophotometer (Hachioji, Japan). The photoluminescence properties were investigated using a Jasco FP-8500 spectrofluorometer (Hachioji, Japan). The X-ray absorption near-edge fine structure (XANES) measurements at the $\mathrm{Ce} L_{3}$-edge were performed at room temperature on a Wiggler beamline 17C at the National Synchrotron Radiation Research Center (NSRRC), Taiwan. The monochromator Si (111) crystals were used in Wiggler beamline $17 \mathrm{C}$. The energy resolution at the Ce $L_{3}$-edge (5723 $\mathrm{eV}$ ) was about $0.4 \mathrm{eV}$. The XANES spectra at the $\mathrm{C}$ K-edge were recorded at beamline 20A using total electron yield (TEY) mode at the National Synchrotron Radiation Research Center (NSRRC), Taiwan. The magnetization was measured at room temperature using a vibrating sample magnetometer (VSM) at the Institute of Physics, Academia Sinica, Taiwan. 


\section{Results and Discussion}

\subsection{Microstructure of the Composites}

Figure 1 presents the XRD pattern of pristine polymer, $\mathrm{CeO}_{2}$ nanoparticles and $\mathrm{CeO}_{2} /$ polymer composites with different amounts of $\mathrm{CeO}_{2}$ nanoparticles. The XRD pattern of $\mathrm{CeO}_{2}$ nanoparticles indicates peaks at $2 \theta$ about $28.5^{\circ}, 33.1^{\circ}, 47.4^{\circ}, 56.3^{\circ}, 59.1^{\circ}, 69.3^{\circ}, 76.7^{\circ}$ and $79.1^{\circ}$ which corresponds to the lattice plane of (111), (200), (220), (311), (222), (400), (331) and (420) of $\mathrm{CeO}_{2}$ with space group Fm $\overline{3} m$ (JCPDS file No. 34-0394). No additional peaks corresponding to other phases were observed which confirms the phase purity of the sample. In the pristine polymer, a relatively broad peak centered at $2 \theta$ equal to $19.8^{\circ}$ was observed, indicating the amorphous nature of the polymer. Moreover, the XRD pattern of $\mathrm{CeO}_{2} /$ polymer composites contains all peaks from both components which indicates that the efficient blend of two components.

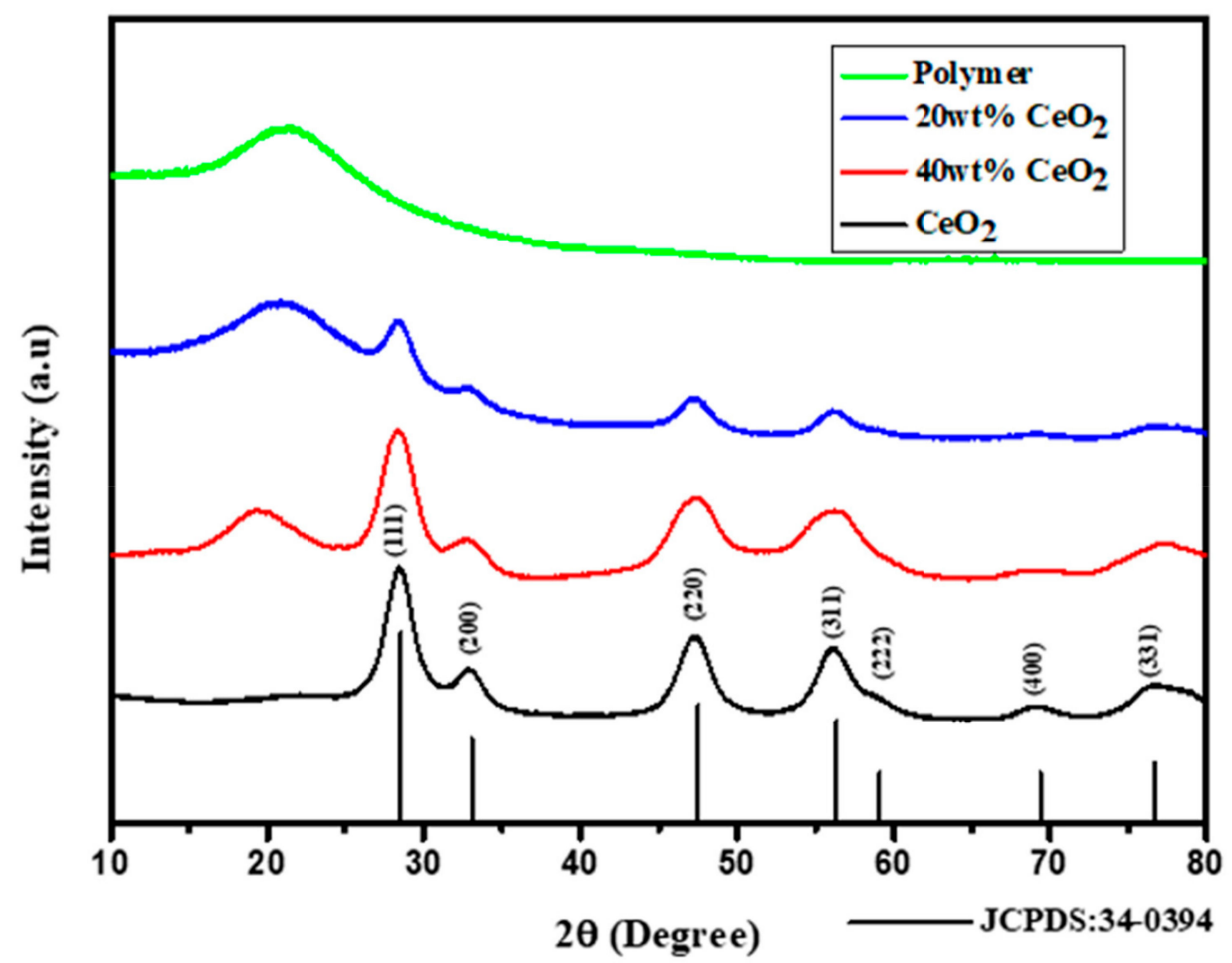

Figure 1. X-ray diffraction (XRD) pattern of polymer composite with $20 \mathrm{wt} \%$ and $40 \mathrm{wt} \% \mathrm{CeO}_{2}$ nanoparticles (NPs).

Figure 2 shows TEM images of $\mathrm{CeO}_{2}$ nanoparticles and $\mathrm{CeO}_{2} /$ polymer composites. As can be seen from Figure $2 \mathrm{a}$, the $\mathrm{CeO}_{2}$ nanoparticles highly aggregated together due to the small size and Van der Waal's forces. The size of the cluster is as large as the micron scale. According to selected area electron diffraction (SAED) analysis (inset of Figure 2a), the particles can be identified as $\mathrm{CeO}_{2}$. Figure $2 \mathrm{~b}$ represents the high-resolution TEM (HRTEM) observation, the particles are highly crystallized with the $\mathrm{d}$-spacing of $0.31 \mathrm{~nm}$ which is consistent with (111) plane of $\mathrm{CeO}_{2}$ nanoparticles. After the composite formation, the aggregation was suppressed. Figure $2 \mathrm{c}, \mathrm{d}$ is the TEM and HRTEM images of composites in which the weight ratio between $\mathrm{CeO}_{2}$ and polymer is 0.4 , showing that the $\mathrm{CeO}_{2}$ cluster size was reduced significantly. As the amount of $\mathrm{CeO}_{2}$ is further increased (weight ratio between $\mathrm{CeO}_{2}$ and polymer is 0.5 ), the degree of dispersion in the polymer is suppressed. The size of the $\mathrm{CeO}_{2}$ cluster was then increased again (Figure S2). 

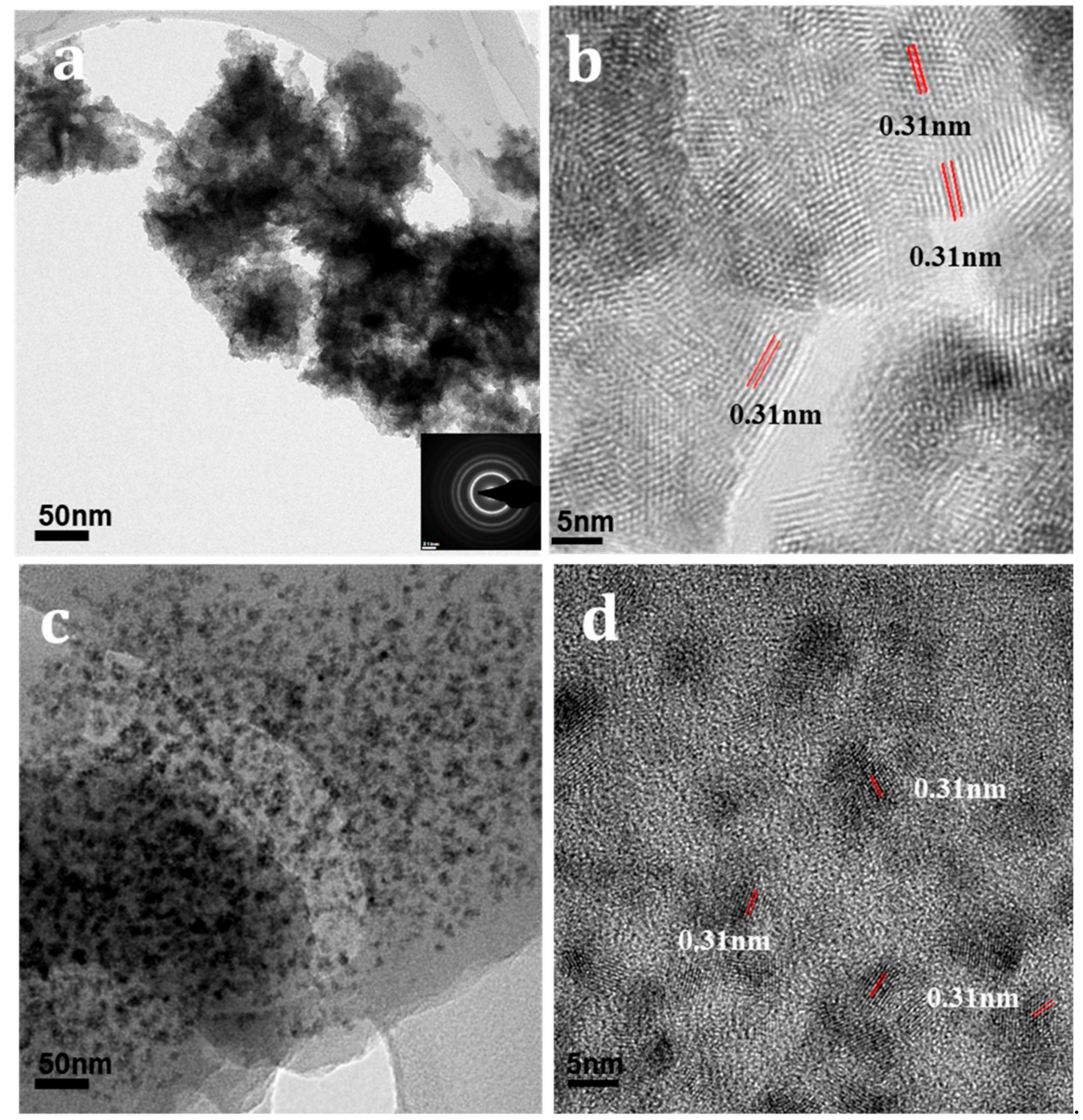

Figure 2. (a) Transmission electron microscope (TEM) image and (b) high-resolution TEM (HRTEM) of $\mathrm{CeO}_{2}$ nanoparticles. (c) TEM image and (d) HRTEM image of polymer composite with $40 \mathrm{wt} \%$ $\mathrm{CeO}_{2}$ nanocomposites.

The decrease in the $\mathrm{CeO}_{2}$ cluster size could be attributed to the interaction between $\mathrm{CeO}_{2}$ nanoparticles and polymer. As mentioned earlier, the polymer used here tends to attract electrons. It has been predicted that naphthalene bisimide units of the polymer are electron-deficient moieties that accept an electron from spirobiflourene moieties [21]. Due to the electron-withdrawing character of the imide groups in the polymer, an electron will be withdrawn from $\mathrm{CeO}_{2}$ to the naphthalene bisimide moieties of the polymer. Consequently, the hybridization between oxygen atoms at the top surface and the neighboring cerium atoms will be suppressed. Importantly, the difference in the hybridization between oxygen and cerium can be identified easily by the change in the Ce valence. $X$-ray absorption spectroscopy (XAS) is a unique method that provides electronic structural information on the orbital symmetry and spin state of the materials and was thus utilized to determine the $C$ hybridization as well as the Ce charge statecaused by the composite formation.

The C K-edge XANES of the composites are shown in Figure 3. The main peak at $287.2 \mathrm{eV}$ (peak A) is attributed to the transitions from $C 1$ s to the unoccupied states of $C=O \pi^{*}$ characters. The peak intensity reflects the unoccupied state of the $\pi^{*}$ character. After the attachment of $\mathrm{CeO}_{2}$, the polymer peak intensity decreased significantly, indicating that more electrons transfer into the polymer. As for the peak located at $290.4 \mathrm{eV}$ (peak B), which correspond to carbon atoms in polymer attached to hydrogen, nitrogen or other species, shows a clear increase. This suggests that the interfacial $\mathrm{C}-\mathrm{O}-\mathrm{Ce}$ bonding is formed for the $\mathrm{CeO}_{2} /$ polymer composites. 


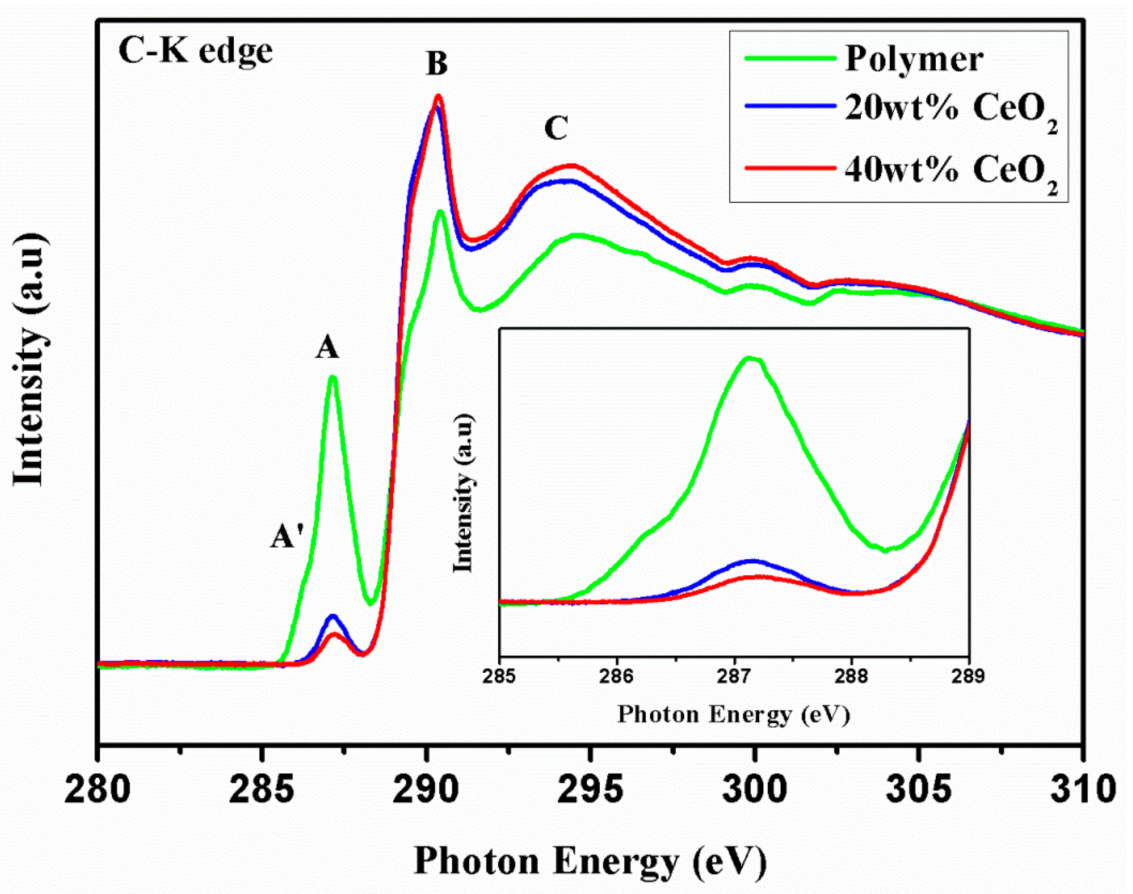

Figure 3. C-K edge X-ray absorption near-edge fine structure (XANES) of polymer, polymer composite with $20 \mathrm{wt} \%$ and $40 \mathrm{wt} \% \mathrm{CeO}_{2}$ nanoparticles (inset figure shows the magnified peak $\mathrm{A}$ ).

The variation in the $\mathrm{Ce}$ charge state in ceria between the composites and pure $\mathrm{CeO}_{2}$ was investigated using XANES of the Ce- $L$ edge. The normalized XANES spectra of $C e L_{3}$ edge of composites containing different ceria nanoparticle concentration are shown in Figure 4. To determine the Ce valance, an arctangent function was subtracted from each to exclude the edge jump. The XANES spectra of each sample were then fitted with five Gaussian functions. The background subtraction method and assignment of peaks was reported in previous literature [22]. Among these, the shoulder-like feature at about $5727-5729 \mathrm{eV}$ (component $\mathrm{C}$ ) is contributed by the trivalent $\mathrm{Ce}$ (in a final $4 \mathrm{f}^{1}(5 \mathrm{~d} 6 \mathrm{~s})^{4}$ state). Therefore, the $\mathrm{Ce}^{3+}$ concentration in the $\mathrm{CeO}_{2}$ matrix $\left(\mathrm{Ce}^{3+} /\left(\mathrm{Ce}^{3+}+\mathrm{Ce}^{4+}\right)\right)$ can be then expressed as the ratio $C^{C e^{3+}}=\frac{I_{C}}{I_{T}}$. Where $\mathrm{I}_{\mathrm{C}}$ is the deconvoluted peak $\mathrm{C}$ intensity and $\mathrm{I}_{\mathrm{T}}$ is the intensity sum of peaks $\mathrm{A}, \mathrm{B}$ and $\mathrm{C}$ [23]. According to this estimation, the $\mathrm{Ce}^{3+}$ concentration (the ratio of the concentration of $\left.\mathrm{Ce}^{3+} /\left(\mathrm{Ce}^{3+}+\mathrm{Ce}^{4+}\right)\right)$ was about $10 \%$ in the raw $\mathrm{CeO}_{2}$ nanoparticles. For the composite, whereas the weight ratio between $\mathrm{CeO}_{2}$ and polymer is 0.2 and 0.4 , the $\mathrm{Ce}^{3+}$ concentration decreases to $7.5 \%$ and $7 \%$, respectively. The decrease in the $\mathrm{Ce}^{3+}$ concentration confirms that electrons transfer from $\mathrm{CeO}_{2}$ into the polymer.

The above XANES analysis of the $\mathrm{C} K$ edge and Ce $L$ edge demonstrated the charge transfer between $\mathrm{CeO}_{2}$ nanoparticles and the polymer, which results in the increase in electrons in the $\mathrm{C}=\mathrm{O}$ of polymer and the decrease of hybridization between $\mathrm{Ce}$ and $\mathrm{O}$ in $\mathrm{CeO}_{2}$ nanoparticles. This implies that more $\mathrm{Ce}^{3+}$ was induced at the particle surface. It is worth noting that among composites with different amounts of $\mathrm{CeO}_{2}$ nanoparticles, the decrease in the $\mathrm{Ce}^{3+}$ concentration is almost the same. This suggests that the interaction occurs only at the $\mathrm{CeO}_{2}$ nanoparticle surface. 


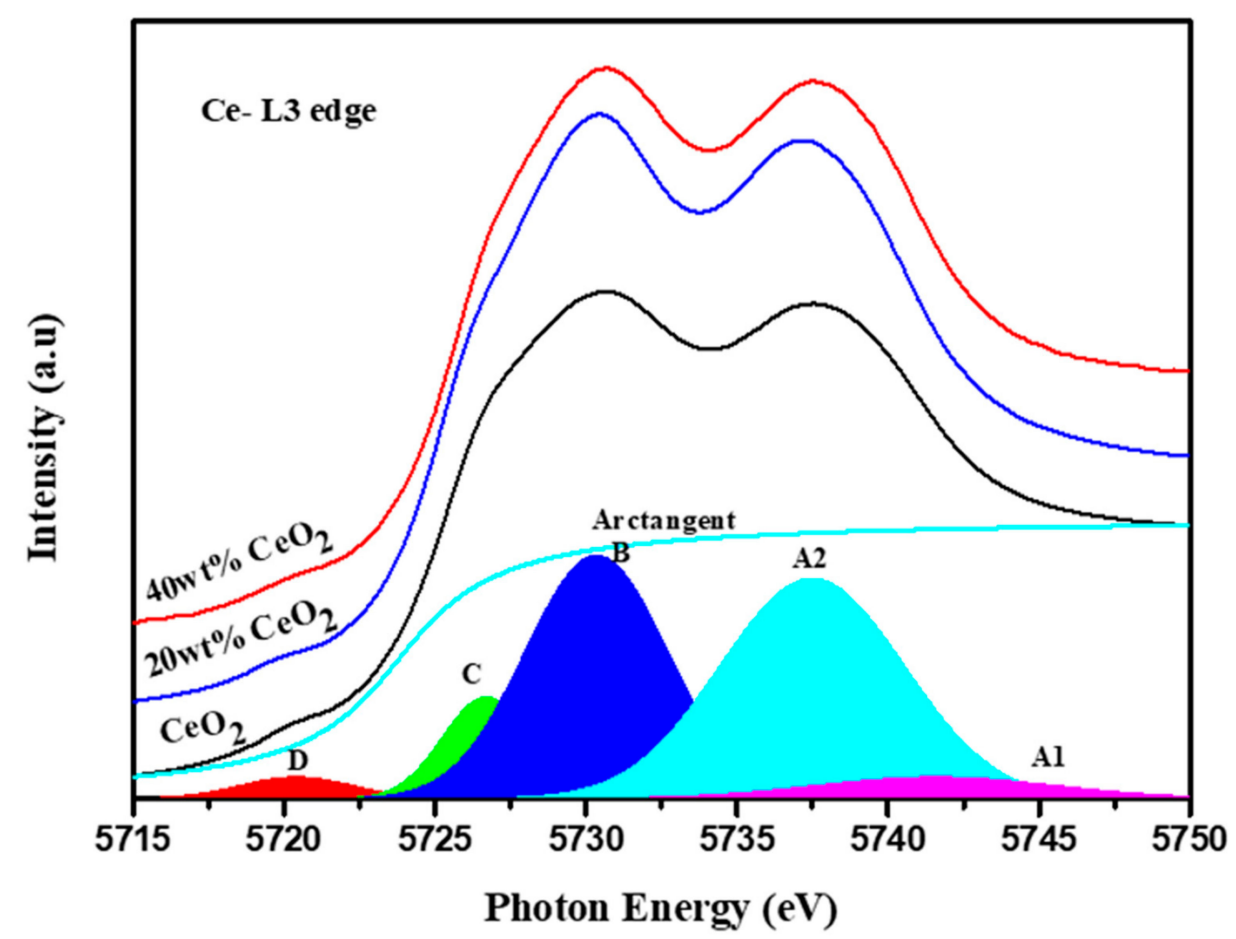

Figure 4. XANES of $\mathrm{Ce} \mathrm{L}$ edge of $\mathrm{CeO}_{2}$, polymer composite with $20 \mathrm{wt} \%$ and $40 \mathrm{wt} \% \mathrm{CeO}_{2}$ nanoparticles.

\subsection{Optical Properties of the Composites}

The original characteristics of both components would be affected by the change in the electronic structure as mentioned above. The UV-Vis spectra of the ceria, polymer and composites are shown in Figure 5. The maximum absorption peak is observed at $350 \mathrm{~nm}$ for the ceria nanoparticles which arise from the electron transition from the $2 p$ valence band $(\mathrm{VB})$ of $\mathrm{O}^{2-}$ to the $4 f$ conduction band (CB) of $\mathrm{Ce}^{4+}$ in $\mathrm{CeO}_{2}$. For the polymer, a broad absorption band was observed possibly due to the $\pi-\pi^{*}$ transition and the intramolecular charge transfer between spirobifluorenes and naphthalene bisimides [21]. It should be noted that the absorption band of the composites is broader than that of the polymer. When polymer is incorporated with $\mathrm{CeO}_{2}$ nanoparticles the polymer absorption is enhanced and the absorption edge shifts to the longer wavelength. This broad absorption and longer absorption edge can be attributed to the charge transfer from the ceria nanoparticles into naphthalene bisimide moieties of the polymer as demonstrated by XAS analysis in the previous section.

The optical band gap can be determined using Tauc's model using the UV-Vis absorption spectra. Tauc's model is given by:

$$
\alpha h v=a\left(h v-E_{g}\right)^{n}
$$

where $\alpha$ is absorption coefficient, $h v$ is photon energy, $a$ is a constant and $n$ is the index characterizing the nature of electronic transition causing optical absorption. $n$ can take on values of 3,2,3/2, or 1/2, corresponding to indirect prohibited, indirect permitted, directly prohibited, and directly permitted transitions, respectively. A graph plotted between $(\alpha h v)^{1 / n}$ as ordinate and hv as abscissa. The extrapolation of the linear part of the graph to $(\alpha h v)^{1 / n}=0$ (Tauc's plot) gives the optical band gap. Accordingly, the optical band gap is $2.97,2.02$ and $1.70 \mathrm{eV}$ for $\mathrm{CeO}_{2}$ nanoparticles, polymer, and composite in which the ratio between $\mathrm{CeO}_{2}$ to polymer is 0.4 , respectively. The change in the composite band gap indicates the intramolecular charge transfer between spirobifluorenes and naphatalene naphthalene bisimides that leads to a change in the band structure. 

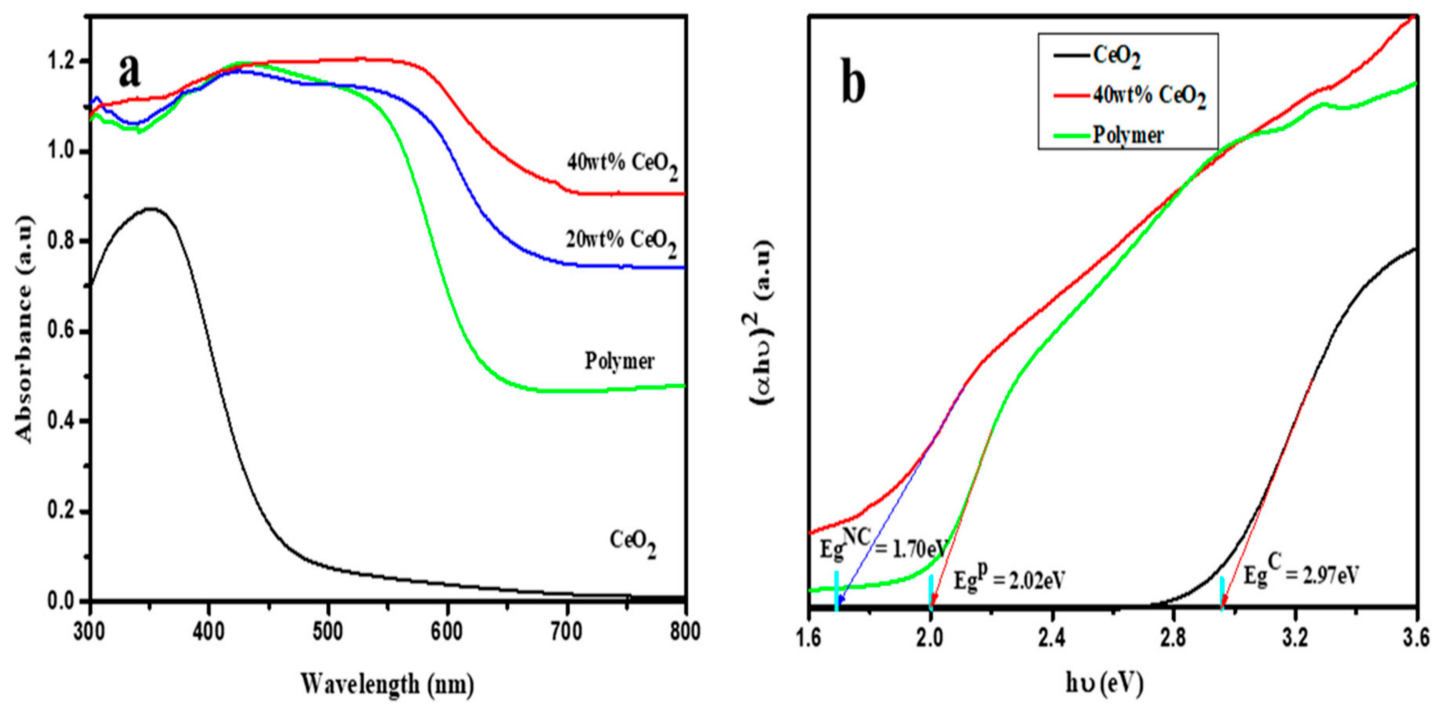

Figure 5. (a) Ultraviolet-visible (UV-Vis) spectra of $\mathrm{CeO}_{2}$, polymer, polymer composite with $20 \mathrm{wt} \%$ and $40 \mathrm{wt} \% \mathrm{CeO}_{2}$ nanoparticles. The determination of band gap is shown in (b).

Photoluminescence (PL) is a remarkable method to probe certain structural aspects and provide information at short and medium-range, where the degree of local order such as structurally inequitable sites can be distinguished by their different types of electronic transitions and are linked to specific structural arrangements. The structural and electronic order or disorder effects and the nature of bonding in a conjugated polymer have a key impact on the optical property. Figure 6 shows the room-temperature PL spectra of the studied materials excited at $441 \mathrm{~nm}$. No emission peak for $\mathrm{CeO}_{2}$ nanoparticles can be observed. A broad emission band from $550 \mathrm{~nm}$ to $750 \mathrm{~nm}$ with a maximum emission peak $(631 \mathrm{~nm})$ was observed for the polymer. The excitons were created by photoexcitation in which the holes and electrons are located in the highest occupied molecular orbital (HOMO) and lowest unoccupied molecular orbital (LUMO) of the polymer, respectively. The electron and hole recombination is responsible for this emission peak. After the composite formation, the fluorescence quenching, as well as red shifting of the emission maximum, is observed. The fluorescence quenching may indicate the intramolecular charge transfer that takes place between the two species as has been confirmed by XANES. The emission peak of the composites shifted from $631 \mathrm{~nm}$ to $643 \mathrm{~nm}$. This redshift suggests that there is a change in the polymer electronic structure as well as the chemical environment, which was demonstrated by UV-Vis spectra and XANES of the C-K edge of the polymer. This shift may arise due to the reduction in bandgap and the variation in the interface between the $\mathrm{CeO}_{2}$ nanoparticles and the polymer. 

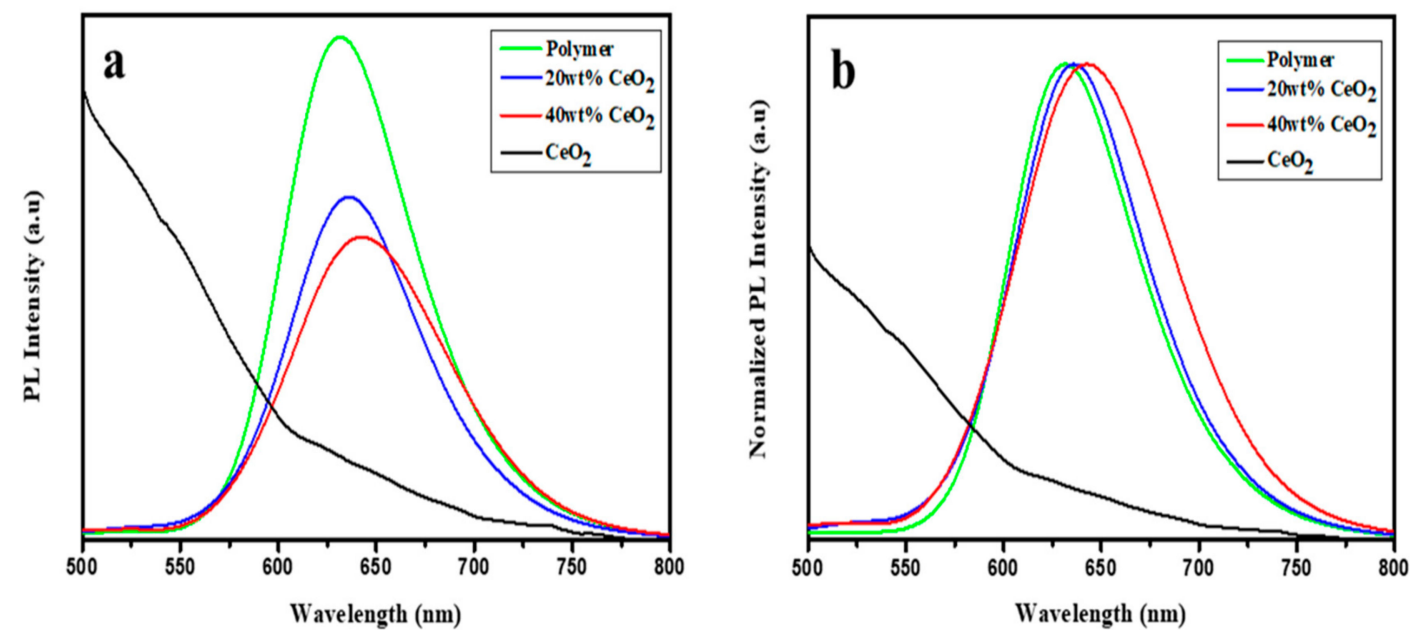

Figure 6. (a) The photoluminescence emission spectra and (b) the normalized spectra of $\mathrm{CeO}_{2}$, polymer, polymer composite with $20 \mathrm{wt} \%$ and $40 \mathrm{wt} \% \mathrm{CeO}_{2}$ nanoparticles.

\subsection{Magnetic Property of the Composites}

M-H curves of the composites (polymer with $40 \mathrm{wt} \%$ ceria NPs), pristine polymer and pure ceria NPs measured at room temperature were shown together in Figure 7. All samples are ferromagnetic. The saturation magnetization value $\left(\mathrm{M}_{\mathrm{s}}\right)$ of the $\mathrm{CeO}_{2}$ is $0.008 \mathrm{emu} / \mathrm{g}$ (inset in Figure 7), which is close to those in the literature. In the ceria, the ferromagnetism origin is attributed to the presence of defects, including the cerium vacancy and oxygen vacancy in the structure [24,25]. Several models have been proposed to explain the exchange mechanism [26-28]. As for the polymer, the value of $\mathrm{M}_{\mathrm{s}}$ was as high as $0.3 \mathrm{emu} / \mathrm{g}$. The ferromagnetism of the polymer may be due to the formation of delocalized unpaired electrons on the conjugated polymer owing to the topology. These unpaired electrons make spin-spin interactions during charge transfer between the spirobifluorene and naphthalene bisimide. This interaction may be the cause of the ferromagnetism in the polymer. The large value of $H_{c}$ in the polymer can be related to the topology.

In the polymer composite with $40 \mathrm{wt} \% \mathrm{CeO}_{2} \mathrm{NPs}$, the value of $\mathrm{M}_{\mathrm{s}}$ was $0.2 \mathrm{emu} / \mathrm{g}$. It is lower than that of pure polymer. According to the magnetic behavior of pristine polymer and ceria NPs, the decrease in magnetization could be attributed to the lesser content in the polymer. However, it is worth mentioning that the $\mathrm{M}_{\mathrm{s}}$ of composite is higher than the linear combination of both components, demonstrating the magnetism of both components changed by interface interactions. For ceria NPs, it has been demonstrated that within a wide range of defect concentrations, the value of $\mathrm{M}_{\mathrm{S}}$ is proportional to the $\mathrm{Ce}^{3+}$ concentration at the surface. As shown in the previous section, the $\mathrm{Ce}^{3+}$ concentration is less after the composite formation. Based on the above results, the decrease in $\mathrm{CeO}_{2}$ ferromagnetic contribution is predicted. In other words, the polymer magnetic response became stronger in the composite. Both polymer conjugation and delocalization was affected by the electron transfer. Similar results were reported by B. Yang et al. They observed that the saturation ferromagnetism of polymer P3HT mixed with phenyl-C61-butryic acid methyl ester (PCBM) was about $0.65 \mathrm{emu} / \mathrm{g}$ and suggested that the ferromagnetism origin is associated with $\mathrm{P} 3 \mathrm{HT}$ crystallization and the charge transfer between P3HT and PCBM [29]. Accordingly, this study indicated that the magnetism of polymer composite could be further tuned by adjusting the content of each component, and also the interaction at the interface. 


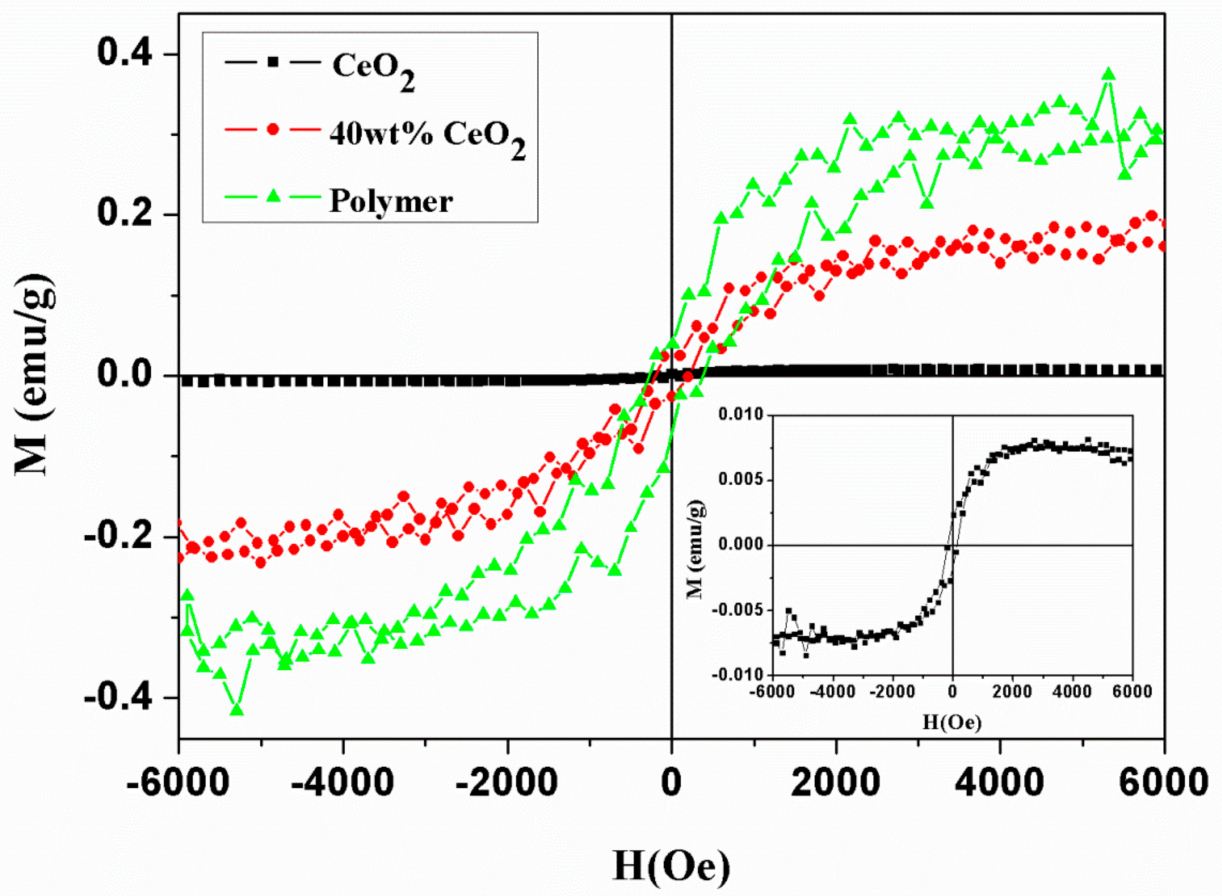

Figure 7. $\mathrm{M}-\mathrm{H}$ curves of $\mathrm{CeO}_{2}$, polymer and polymer composite with $40 \mathrm{wt} \% \mathrm{CeO}_{2}$ nanoparticles. The magnified $\mathrm{M}-\mathrm{H}$ curve of $\mathrm{CeO}_{2}$ is shown in the inset.

\section{Conclusions}

Polymer composite with $\mathrm{CeO}_{2}$ NPs was successfully produced using an ex situ preparation method. This method has an advantage over the in-situ method as it is free from the unnecessary solid remaining in the hybrid and it is simple and used for large-scale production. TEM and XAS analysis results showed that the nanoparticles are well dispersed in the polymer matrix with charge transfer that occurred between the two components. The difference in band structure resulted in a broader absorption peak than the individual components UV-Vis spectra. Photoluminescence revealed the fluorescence quenching and red shifting of the composite peak compared to that of the pristine polymer. The difference in electron hybridization and localization also affected the magnetic response of both components. The magnetization of the pristine polymer was found to be enhanced.

Supplementary Materials: The following are available online at http://www.mdpi.com/2079-4991/9/11/1534/s1, Figure S1: The schematic picture of the polymer synthesis procedure. The chemical formulas of polymers are also shown. Figure S2: TEM investigation of polymer composite with different amount of $\mathrm{CeO}_{2}$, (a) bright image of composite with $20 \mathrm{wt} \% \mathrm{CeO}_{2}$, (b) bright image of composite with $50 \mathrm{wt} \% \mathrm{CeO}_{2}$, (c) HRTEM of composite with $20 \mathrm{wt} \% \mathrm{CeO}_{2}$, (d) HRTEM of composite with $50 \mathrm{wt} \% \mathrm{CeO}_{2}$. The insert figures were corresponding diffraction patterns. Aggregation of nanoparticles was observed in composite with $50 \mathrm{wt} \% \mathrm{CeO}_{2}$.

Author Contributions: S.Y.C. and C.-Y.Y. supervised the experiments and characterization. T.S.B. designed and carried out the experiments and write the manuscript. E.N.T. helped to perform and analyze the results of the TEM. Y.-C.C. and P.-K.H. helped to perform and analyze XAS and VSM results. S.-Y.C. and C.-Y.Y. contributed to writing and editing the manuscript, and all authors contributed to the data analysis and discussion of the results. All authors read and approved the final manuscript.

Funding: This research was funded by the Ministry of Science and Technology, Taiwan (Grant No. MOST 107-2112-M-011-002-MY3). The APC was funded by the Ministry of Science and Technology, Taiwan (Grant No. MOST 107-2112-M-011-002-MY3).

Acknowledgments: The authors acknowledge financial support from the Ministry of Science and Technology, Taiwan.

Conflicts of Interest: The authors declare that they have no competing interests. 


\section{Abbreviations}

NP: nanoparticles; RAD: relaxative auto-dispersion; EG: ethylene glycol; THF: tetrahtdofuran; XRD: x-ray diffractometer; NSRRC: national synchrotron research center; TEM: transmission electron microscope; SAED: selected area electron diffraction; HRTEM: high resolution transmission electron microscope; XAS: X-ray absorption spectroscopy; XANES: $x$-ray absorption near edge spectroscopy; TEY: total electron yield; UV-Vis: ultraviolet-visible; VSM: vibrating sample magnetometer; CB: conduction band; VB: valence band; PL: photoluminescence; HOMO: highest occupied molecular orbital; LUMO: lowest unoccupied molecular orbital; P3HT: poly(3-hexylthiophene); PCBM: phenyl-C61-butryic acid methyl ester.

\section{References}

1. Bellucci, S.; Bolesta, I.; Guidi, M.C.; Karbovnyk, I.; Lesivciv, V.; Micciulla, F.; Pastore, R.; Popov, A.I.; Velgosh, S. Cadmium clusters in CdI2 layered crystals: The influence on the optical properties. J. Phys. Condens. Matter 2007, 19, 395015. [CrossRef]

2. Song, S.; Kotobuki, M.; Zheng, F.; Xu, C.; Savilov, S.V.; Hu, N.; Lu, L.; Wang, Y.; Li, W.D.Z. A hybrid polymer/oxide/ionic-liquid solid electrolyte for Na-metal batteries. J. Mater. Chem. A 2017, 5, 6424-6431. [CrossRef]

3. Velayutham, P.; Sahu, A.; Parthasarathy, S. A Nafion-Ceria Composite Membrane Electrolyte for Reduced Methanol Crossover in Direct Methanol Fuel Cells. Energies 2017, 10, 259. [CrossRef]

4. Mohanapriya, M.K.; Deshmukh, K.; Ahamed, M.B.; Chidambaram, K.; Khadheer Pasha, S.K. Influence of Cerium Oxide (CeO2) Nanoparticles on the Structural, Morphological, Mechanical and Dielectric Properties of PVA/PPy Blend Nanocomposites. Mater. Today Proc. 2016, 3, 1864-1873. [CrossRef]

5. Zhang, L.; Chen, S.; Yuan, S.; Wang, D.; Hu, P.-H.; Dang, Z.-M. Low dielectric loss and weak frequency dependence of dielectric permittivity of the CeO2/polystyrene nanocomposite films. Appl. Phys. Lett. 2014, 105, 052905. [CrossRef]

6. Li, Y.; Zhang, J.; Ni, X.; Wang, L.; Yang, C. Facile fabrication of raspberry-like polystyrene/ceria composite particles and their catalytic application. Colloids Surfaces A 2018, 538, 818-824. [CrossRef]

7. Sabitha, G.; Reddy, K.B.; Yadav, J.S.; Shailaja, D.; Sivudu, K.S. Ceria/vinylpyridine polymer nanocomposite: An ecofriendly catalyst for the synthesis of 3,4-dihydropyrimidin-2(1H)-ones. Tetrahedron Lett. 2005, 46, 8221-8224. [CrossRef]

8. Ecco, L.G.; Fedel, M.; Ahniyaz, A.; Deflorian, F. Influence of polyaniline and cerium oxide nanoparticles on the corrosion protection properties of alkyd coating. Prog. Org. Coat. 2014, 77, 2031-2038. [CrossRef]

9. Eduok, U.; Jossou, E.; Tiamiyu, A.; Omale, J.; Szpunar, J. Ceria/Acrylic Polymer Microgel Composite: Synthesis, Characterization, and Anticorrosion Application for API 5L X70 Substrate in Chloride-Enriched Medium. Ind. Eng. Chem. Res. 2017, 56, 5586-5597. [CrossRef]

10. Park, S.; Park, C.; Yoon, H. Chemo-Electrical Gas Sensors Based on Conducting Polymer Hybrids. Polymers 2017, 9, 155. [CrossRef]

11. Ansari, A.A.; Khan, M.A.M.; Khan, M.N.; Alrokayan, S.A.; Alhoshan, M.; Alsalhi, M.S. Optical and electrical properties of electrochemically deposited polyaniline/CeO2hybrid nanocomposite film. J. Semicond. 2011, 32, 043001. [CrossRef]

12. Itoh, T.; Uchida, T.; Izu, N.; Matsubara, I.; Shin, W. Effect of Core-Shell Ceria/Poly(vinylpyrrolidone) (PVP) Nanoparticles Incorporated in Polymer Films and Their Optical Properties. Materials 2013, 6, 2119-2129. [CrossRef] [PubMed]

13. Aguirre, M.; Paulis, M.; Leiza, J.R. UV screening clear coats based on encapsulated CeO2 hybrid latexes. J. Mater. Chem. A 2013, 1, 3155. [CrossRef]

14. Aklalouch, M.; Calleja, A.; Granados, X.; Ricart, S.; Boffa, V.; Ricci, F.; Puig, T.; Obradors, X. Hybrid sol-gel layers containing $\mathrm{CeO} 2$ nanoparticles as UV-protection of plastic lenses for concentrated photovoltaics. Sol. Energy Mater. Sol. Cells 2014, 120, 175-182. [CrossRef]

15. Liu, K.-Q.; Kuang, C.-X.; Zhong, M.-Q.; Shi, Y.-Q.; Chen, F. Synthesis, characterization and UV-shielding property of polystyrene-embedded CeO2 nanoparticles. Opt. Mater. 2013, 35, 2710-2715. [CrossRef]

16. Guo, Q.; Ghadiri, R.; Weigel, T.; Aumann, A.; Gurevich, E.L.; Esen, C.; Medenbach, O.; Cheng, W.; Chichkov, B.; Ostendorf, A. Comparison of in Situ and ex Situ Methods for Synthesis of Two-Photon Polymerization Polymer Nanocomposites. Polymers 2014, 6, 2037-2050. [CrossRef] 
17. Da Silva, F.C.; Pinheiro da Silva, M.F.; Briotto Filho, D.; José Politi, M.; Bacani, R.; Rezende Triboni, E. Tuned charge-transfer between ceria and 1,4,5,8-naphthaldiimide as a function of pH. J. Lumin. 2018, 194, 240-247. [CrossRef]

18. Dahle, J.T.; Arai, Y. Environmental geochemistry of cerium: Applications and toxicology of cerium oxide nanoparticles. Int. J. Environ. Res. Public Health 2015, 12, 1253-1278. [CrossRef]

19. Masui, T.; Machida, K.; Sakata, T.; Mori, H.; Adachi, G. Preparation and characterization of cerium oxide ultrafine particles dispersed in polymer thin films. J. Alloys Compd. 1997, 256, 97-101. [CrossRef]

20. Wang, W.; Zhang, B.; Jiang, S.; Bai, H.; Zhang, S. Use of CeO(2) Nanoparticles to Enhance UV-Shielding of Transparent Regenerated Cellulose Films. Polymers 2019, 11, 458. [CrossRef]

21. Yu, C.Y.; Hsu, Y.-J. Synthesis, characterization, optical and electrochemical properties of spirobifluorene based polymers containing electron deficient moieties. Polymer 2016, 84, 65-71. [CrossRef]

22. Nachimuthu, P.; Shih, W.-C.; Liu, R.-S.; Jang, L.-Y.; Chen, J.-M. The Study of Nanocrystalline Cerium Oxide by X-Ray Absorption Spectroscopy. J. Solid State Chem. 2000, 149, 408-413. [CrossRef]

23. Lee, W.; Chen, S.-Y.; Chen, Y.-S.; Dong, C.-L.; Lin, H.-J.; Chen, C.-T.; Gloter, A. Defect Structure Guided Room Temperature Ferromagnetism of Y-Doped CeO2 Nanoparticles. J. Phys. Chem. C 2014, 118, 26359-26367. [CrossRef]

24. Liu, Y.; Lockman, Z.; Aziz, A.; MacManus-Driscoll, J. Size dependent ferromagnetism in cerium oxide (CeO2) nanostructures independent of oxygen vacancies. J. Phys. Condens Matter 2008, 20, 165201. [CrossRef]

25. Li, M.; Ge, S.; Qiao, W.; Zhang, L.; Zuo, Y.; Yan, S. Relationship between the surface chemical states and magnetic properties of $\mathrm{CeO} 2$ nanoparticles. Appl. Phys. Lett. 2009, 94, 152511. [CrossRef]

26. Coey, J.M.D.; Venkatesan, M.; Fitzgerald, C.B. Donor impurity band exchange in dilute ferromagnetic oxides. Nat. Mater. 2005, 4, 173-179. [CrossRef]

27. Coey, J.M.D.; Stamenov, P.; Gunning, R.; Venkatesan, M.; Paul, K. Ferromagnetism in defect-ridden oxides and related materials. N. J. Phys. 2010, 12, 053025. [CrossRef]

28. Coey, M.; Ackland, K.; Venkatesan, M.; Sen, S. Collective magnetic response of CeO2 nanoparticles. Nat. Phys. 2016, 12, 694. [CrossRef]

29. Yang, B.; Xiao, Z.; Yuan, Y.; Jayaraman, T.V.; Shield, J.E.; Skomski, R.; Huang, J. Room-temperature organic ferromagnetism in the crystalline poly(3-hexylthiophene): Phenyl-C61-butyric acid methyl ester blend film. Polymer 2013, 54, 490-494. [CrossRef]

(C) 2019 by the authors. Licensee MDPI, Basel, Switzerland. This article is an open access article distributed under the terms and conditions of the Creative Commons Attribution (CC BY) license (http://creativecommons.org/licenses/by/4.0/). 